\title{
C*-Álgebras e a Descrição da Mecânica Quântica
}

\author{
$\mathrm{C}^{*}$-Algebras and the Description of Quantum Mechanics
}

\author{
Sergio Floquet*1, Augusto A. de Castro Júnior ${ }^{2}$, Marco A. S. Trindade ${ }^{3}$, J. David M. Vianna ${ }^{4,5}$ \\ ${ }^{1}$ Universidade Federal do Vale do São Francisco, Colegiado de Engenharia Civil, Juazeiro, BA, Brasil \\ ${ }^{2}$ Universidade Federal da Bahia, Instituto de Matemática, Salvador, BA, Brasil \\ ${ }^{3}$ Universidade do Estado da Bahia, Departamento de Ciências Exatas e da Terra, Laboratório de Física, Salvador, BA, Brasil \\ ${ }^{4}$ Universidade Federal da Bahia, Instituto de Física, Salvador, BA, Brasil \\ ${ }^{5}$ Universidade de Brasília, Instituto de Física, Brasília, DF, Brasil
}

Recebido em 05 de Setembro, 2017. Revisado em 10 de Novembro, 2017. Aceito em 18 de Novembro, 2017.

\begin{abstract}
Neste artigo apresentamos a Teoria das $\mathrm{C}^{*}$-Álgebras. Demonstramos dois importantes resultados que são: o Teorema de Gelfand, que associa toda $C^{*}$-Álgebra Abeliana às funções contínuas sobre um espaço Hausdorff compacto, e o Teorema de Gelfand-Neumark, que relaciona toda $C^{*}$-Álgebra não Abeliana aos operadores lineares sobre um espaço de Hilbert. Em seguida, mapeamos a Mecânica Clássica dentro da teoria da C*-Álgebra, obtendo uma prescrição algébrica para os estados e às observáveis clássicas. Ao estendermos essa construção para o caso Quântico, preservando a prescrição algébrica e utilizando o Princípio da Incerteza, obtemos que os estados quânticos devem ser descritos por vetores do Espaço de Hilbert enquanto que os observáveis quânticos são os operadores auto-adjuntos sobre este espaço; discutimos brevemente sobre a Álgebra de Weyl.
\end{abstract}

Palavras-chave: Mecânica Quântica, C*-Álgebras, Teorema de Gelfand-Neumark.

In this paper we present the theory of $\mathrm{C}^{*}$-Algebras. We show two major results that are Gelfand's Theorem, which associates every Abelian $\mathrm{C}^{*}$-Algebra as continuous functions on a compact Hausdorff space, and the Gelfand-Neumark Theorem, which relates all non-Abelian $\mathrm{C}^{*}$-Algebra to linear operators on a Hilbert space. Then we map the Classical Mechanics into the $\mathrm{C}^{*}$-Algebra's theory, obtaining an algebraic prescription for classical states and observables. When we extend this construction to the Quantum case, preserving the algebraic prescriptions and using the Uncertainty Principle, we obtain that quantum states must be described by vectors in a Hilbert space while quantum observables are the self-adjoint operators on this space; we briefly discussed the Weyl's Algebra.

Keywords: Quantum Mechanics, C*-Algebras, Gelfand-Neumark Theorem.

\section{Introdução}

Uma das inquietudes iniciais de quase todos os estudantes ao se depararem pela primeira vez com a Mecânica Quântica é a sua descrição Matemática. Enquanto os estados na Mecânica Clássica são tipicamente vetores que guardam os dados de posição e momento de cada partícula do sistema (portanto, elementos de um espaço vetorial de dimensão finita), enquanto as observáveis são funções nesses espaços (como temperatura, pressão e energia, por exemplo), na Mecânica Quântica temos um modelo bem mais sofisticado. A necessidade de um Espaço de Hilbert para descrever os estados e de operadores auto-adjuntos para caracterizar os observáveis, quebra intuitivamente a noção clássica onde os estados e observáveis são elementos de um espaço de fase e funções contínuas sobre esse espaço, respectivamente. $\mathrm{O}$ presente artigo tem como objetivo mostrar que, se tomarmos a descrição da Mecânica Clássica baseada nas $\mathrm{C}^{*}$-Álgebras, podemos obter que a descrição Matemática

*Endereço de correspondência: sergio.floquet@univasf.edu.br para o caso Quântico é naturalmente dada por operadores auto-adjuntos sobre um espaço de Hilbert, fazendo uso somente do Princípio da Incerteza. Essa abordagem se mostrou mais que profícua a partir da construção GNS (Gelfand-Neumark-Segal). Como muito da teoria Quântica foge à intuição que temos no dia-a-dia, não fica claro que a modelagem dos fenômenos quânticos não pudesse ser feita de formas matemáticas tão diversas que possivelmente levassem a previsões até mesmo contraditórias. A Construção GNS possui a relevância de mostrar que matematicamente quaisquer modelos para a Cinemática da Mecânica Quântica são isomorfos, isto é, são matematicamente equivalentes. Ademais, nos proveu uma estrutura matemática padrão para tais modelos, via $C^{*}-$ Álgebras.

As $C^{*}$-Álgebras originaram-se a partir do estudo das Álgebras de Operadores propostas por John von Neumann $n^{1}$ em 1929 1]. Nascido no império Astro-Húngaro em 1903, von Neumann cunhou o conceito abstrato de

\footnotetext{
${ }^{1}$ Terminou seu doutoramento aos 22 anos, em 1926 tornou-se o Privatdozent (Professor Associado) mais novo da história da Uni-
} 
espaços de Hilbert e desenvolveu a teoria espectral de operadores normais limitados e ilimitados sobre este mesmo espaço [2]. As álgebras estudadas por von Neumann e Murray, atualmente denominadas de Álgebras de von Neumann, são certas sub-álgebras de $B(\mathcal{H})$, a álgebra dos operadores limitados sobre um espaço de Hilbert, que tiveram um papel importante na estruturação matemática da Mecânica Quântica [3 6].

Em 1941, Israel Gelfand 7. combinou o conceito de álgebra de operadores com a teoria de espaços de Banach, resultando na definição de Álgebra de Banach. Ele procedeu formulando uma teoria espectral intrínseca às Álgebras de Banach e provando quase todos resultados básicos na teoria de Álgebras de Banach Abelianas.

Já em 1943, Israel Gelfand e Mark Neumark 2] 8d definiram o que hoje é conhecido como $\mathrm{C}^{*}$-Álgebra e em seguida publicaram um artigo provando o teorema que recebeu seus nomes, Teorema de Gelfand-Neumark, onde se demonstra que toda $C^{*}$-Álgebra não abeliana é isometricamente isomorfa à álgebra de operadores sobre um espaço de Hilbert. Neste artigo proposto por Gelfand e Neumark, já havia as ideias do que conhecemos hoje em dia como sendo a construção GNS (GelfandNeumark-Segal), que conecta estados a representações sobre o espaço de Hilbert. Foi em 1947 que Irving Segal [9] demonstrou a construção GNS e definiu a ideia de estado como um funcional linear positivo e normalizado.

$\mathrm{Na}$ década de 60, a teoria das $\mathrm{C}^{*}$-Álgebras começou a ser utilizada em diversos campos da matemática. Em geral, a estratégia utilizada é tentar codificar uma dada estrutura matemática numa $C^{*}$-Álgebra e a partir disso, obter informações sobre essa estrutura provando teoremas sobre a $C^{*}$-Álgebra associada à estrutura 10 14. $\mathrm{Na}$ Física as $\mathrm{C}^{*}$-Álgebras desempenham um papel importante na formulação da Mecânica Estatística [15, 16], na descrição algébrica na Teoria Quântica de Campos [17, em Geometrias não Comutativas [18] e em Teorias Térmicas [19 23], como a Dinâmica de Campos Térmicos 24.

É importante ressaltar que a construção GNS permite uma interpretação de que o espaço de Hilbert é dependente do estado ou da preparação do sistema físico a ser considerado e não uma característica perene da teoria 25]. Representações distintas dos espaços de Hilbert são necessárias para a descrição de situações físicas diferentes. Dessa forma, a proposta basilar da abordagem algébrica é enfatizar que os objetos primários da teoria são os observáveis (ou os campos, no contexto da teoria quântica de campos) munidos de uma topologia adequada, em vez de considerar um esquema de modelagem que possui como ponto de partida um espaço de Hilbert específico. Por conseguinte os métodos algébricos são capazes de cap-

versidade de Berlim. Entre 1927 e 1929 publicou 32 artigos, quase 1 artigo por mês.

${ }^{2}$ Também conhecido como Naimark, sendo este uma tradução equivocada do russo para o inglês. O próprio autor adota a tradução do seu nome para Neumark, como é possível perceber nas referências originais. turar os elementos essenciais da teoria quântica através de um formalismo matemático consistente e rigoroso.

É com esse espírito que neste artigo pretendemos descrever a estrutura matemática da Mecânica Quântica, isto é, queremos que esta estrutura surja naturalmente como uma consequência dos Teoremas relacionados às C*-Álgebras. Para isso, codificamos a Mecânica Clássica dentro da teoria das $C^{*}$-Álgebra abelianas e encontramos o que seria, do ponto de vista da $\mathrm{C}^{*}$-Álgebra, os estados e as observáveis. Em seguida, fazemos uso do Princípio da Incerteza de Heisenberg, que constata que os observáveis quânticas são não abelianas, o que implica que a $C^{*}$ Álgebra que descreve a Mecânica Quântica deve ser não abeliana. Com isso, encontramos naturalmente que os observáveis da Mecânica Quântica devem ser os operadores auto-adjuntos sobre o espaço de Hilbert, enquanto que os estados são vetores normalizados deste mesmo espaço.

Este artigo tem caráter pedagógico, e foi organizado na seguinte forma. Na seção 2, fazemos uma breve revisão sobre Análise Funcional começando das noções mais básicas de espaço vetorial até resultados interessantes sobre os espaços de Hilbert. Em seguida, discutimos um pouco sobre a Teoria Espectral, que será necessária ao longo deste texto. Na seção 3, abordaremos as C*Álgebras, discutindo alguns resultados básicos. Posteriormente, encontramos alguns resultados profundos dentro desta teoria que são: o Teorema de Gelfand, que caracteriza as C*-Álgebras abelianas; o Teorema de Gelfand-Neumark responsável pela caracterização das $\mathrm{C}^{*}$-Álgebras não abelianas e a construção GNS, que relaciona estados e representações. Já a seção 4 é destinada a aplicação da Teoria de $C^{*}$-Álgebra à Mecânica Quântica. Para isso, fazemos a conexão entre a Mecânica Clássica e as $\mathrm{C}^{*}$-Álgebras abelianas, construindo uma prescrição algébrica para os estados e as observáveis, o que nos permite derivar a estrutura matemática da Mecânica Quântica como uma consequência natural da descrição via uma $C^{*}$-Álgebra. Finalizamos essa seção falando sobre os operadores de Weyl e a $C^{*}$-Álgebra de Weyl. Por fim, apresentamos nossas conclusões e perspectivas na seção 5 e a demonstração dos Teoremas de Gelfand e Gelfand-Neumark no Apêndice A e B respectivamente.

\section{Um Pouco de Análise Funcional e Teoria Espectral}

Nesta seção faremos uma introdução a alguns conceitos e resultados básicos da Análise Funcional e Teoria Espectral. Apesar do campo da Análise Funcional ser vasto e interessante, nos restringiremos a um breve estudo sobre Espaços de Hilbert. Em seguida discutimos alguns aspectos da Teoria Espectral, baseados essencialmente nas referências 26 27]. Para o leitor interessado num estudo mais detalhado sobre tais temas, e na demonstração dos resultados apresentados, indicamos as referências 28,32$]$. 


\subsection{Propriedades Elementares dos Espaço de Hilbert}

A noção de espaço vetorial é bem abrangente e é utilizada largamente dentro da Matemática. Seja $V$ um espaço vetorial sobre um corpo $\mathcal{K}$; consideraremos ao longo de todo o texto esse corpo como sendo o corpo dos números complexos $\mathcal{K}=\mathbb{C}$. Sobre a estrutura de espaço vetorial, podemos introduzir várias aplicações com diversas propriedades, uma delas é o produto interno.

Um produto interno é uma função sesquilinear ${ }^{3}\langle\cdot, \cdot\rangle$ : $V \times V \rightarrow \mathcal{K}$ que nos permite definir noção de ângulo e distância entre dois elementos quaisquer de $V$.

A partir do conceito de produto internq ${ }^{4}$ dizemos que um elemento, $u \in V$ é unitário se $\langle u, u\rangle=1$, isto é, com módulo de uma unidade. Dois elementos, $u, v \in V$ são ortogonais se $\langle u, v\rangle=0$, onde o símbolo $u \perp v$ indica essa propriedade. Do mesmo modo, podemos estender esse conceito para subespaços de $V$, isto é, sejam $E$ e $F$ subespaços vetoriais de $V$, eles são ditos ortogonais, $E \perp F$, se $\langle u, v\rangle=0, \forall u \in E$ e $v \in F$.

Todo produto interno induz naturalmente uma norma, dada por $\|u\|=\sqrt{\langle u, u\rangle}$, que por sua vez induz uma métrica

$$
d(u, v)=\|u-v\|=\sqrt{\langle u-v, u-v\rangle},
$$

assim, A partir de um espaço vetorial com produto interno podemos naturalmente falar de um espaço normado ou de espaço métrico.

Um espaço vetorial normado é dito ser Separável se existe um subconjunto contável denso nesse espaço.

Por subconjunto contável, entendemos por um subconjunto que possui a mesma cardinalidade que o conjunto dos números naturais, enquanto que dizemos que um subconjunto é denso se o seu fecho, ou seja, o menor fechado que o contém, é todo o espaço. Já um espaço métrico $(E, d)$ é dito Completo se toda sequência de Cauchy $\left(x_{n}\right)$, com $x_{n} \in E$, converge a algum ponto de $E$. Por sequência de Cauchy, entendemos uma sequência $\left(x_{n}\right)$ tal que

$$
d\left(x_{n}, x_{m}\right) \rightarrow 0, \quad \text { quando } n, m \rightarrow+\infty .
$$

Um Espaço de Hilbert $\mathcal{H}$ é justamente um espaço vetorial separáve $5^{5}$ completo e com produto interno.

Um resultado que relaciona espaços vetoriais com produto interno, $(X,\langle.,\rangle$.$) , e espaços de Hilbert, \mathcal{H}$, é o que

\footnotetext{
${ }^{3} \mathrm{O}$ radical sesqui vem do grego e significa um e meio. Uma aplicação $\langle\cdot, \cdot\rangle: V \times V \rightarrow \mathcal{K}$ é sesquilinear se é linear em uma entrada e antilinear na outra, ou seja, é aplicado ao elemento do corpo a conjugação complexa.

${ }^{4}$ Notaremos o produto interno de $a$ e $b$ por $\langle a, b\rangle$.

${ }^{5}$ Apesar de nos trabalhos originais de Von Neumann ser requerida a separabilidade na definição do espaço de Hilbert, atualmente esta condição não é mais exigida e os espaços de Hilbert podem ser tanto separáveis quanto não separáveis. Consideraremos aqui a definição do espaço de Hilbert incluindo a separabilidade, similar a de von Neumann, a fim de simplificar algumas demonstrações. A não separabilidade dos espaços de Hilbert desempenha um importante papel em Teoria Quântica de Campos, onde o sistema possui infinitos graus de liberdade 33.
}

afirma que $(X,\langle.,\rangle$.$) é unitariamente equivalente a um$ subespaço denso num espaço de Hilbert $\mathcal{H}$

$$
(X,\langle., .\rangle) \subset \mathcal{H}
$$

tal $\mathcal{H}$ é chamado de completamento de $X$. Além disso, quaisquer dois completamentos de $X$ são unitariamente equivalentes entre si.

Uma das primeiras consequências da estrutura de produto interno é a obtenção de diversas igualdades e desigualdades envolvendo os vetores; entre elas temos a Desigualdade de Cauchy-Schwarz em que $\forall u, v \in \mathcal{H}$ temos que

$$
|\langle u, v\rangle| \leq\|u\|\|v\| .
$$

De modo geral a Análise Funcional é o ramo da Matemática, mais especificamente da Análise, que estuda espaços de dimensão infinita. Nesta área utiliza-se diversos conceitos de Álgebra Linear, um deles que desempenha grande importância é o conceito de operador. Assim,

Operadores Lineares sobre espaços vetoriais $\mathcal{H}_{1}$ e $\mathcal{H}_{2}$ são aplicações $T: \mathcal{H}_{1} \rightarrow \mathcal{H}_{2}$ em que

$T\left(x_{1}+\alpha y_{1}\right)=T\left(x_{1}\right)+\alpha T\left(y_{1}\right), \forall x_{1}, y_{1} \in \mathcal{H}_{1}, \alpha \in \mathbb{C}$.

A partir da norma induzida pelo produto interno, podemos definir que um operador é Limitado se

$$
\|T\|=\sup _{\|x\| \leq 1}\|T x\|<+\infty
$$

onde essa norma de $\mathrm{T}$ é conhecida como a norma do operador. Vale a pena ressaltar que todo operador linear é limitado se, e somente se, é contínuo. O Conjunto de todos os operadores lineares e limitados é denotado por $B\left(\mathcal{H}_{1}, \mathcal{H}_{2}\right) ;$ se $\mathcal{H}_{1}=\mathcal{H}_{2}$ simplificamos a notação para $B(\mathcal{H})$. Esse conjunto é um espaço vetorial, sendo que

para todo operador $T \in B(\mathcal{H})$ existe um único operador, que notaremos por $T^{*}$, tal que

$$
\langle x, T y\rangle=\left\langle T^{*} x, y\right\rangle, \quad \forall x, y \in \mathcal{H} .
$$

Chamaremos o operador $T^{*}$ de Operador Adjunto de T. Se ocorrer de $T T^{*}=T^{*} T$, então T é dito ser um Operador Normal; se $T^{*}=T^{-1}$, T é um Operador Unitário; se $T=$ $T^{*}$ então T é um Operador Auto-adjunto e se $\langle T x, x\rangle \geq$ $0, \forall x \in \mathcal{H}, \mathrm{T}$ é denominado de Operador Positivo.

O espaço vetorial $B(\mathcal{H}, \mathbb{C})$ é chamado de Espaço Dual de $\mathcal{H}$ e é notado por $\mathcal{H}^{*}$. Os elementos de $\mathcal{H}^{*}$ são denominados de Funcionais Lineares.

Para os espaços de Hilbert, existe uma representação de $\mathcal{H}^{*}$ que o identifica naturalmente com $\mathcal{H}$, essa é a Representação de Riesz dada pela aplicação $\gamma: \mathcal{H} \rightarrow \mathcal{H}^{*}$, $\operatorname{com} \gamma(\xi)=f_{\xi}$, que para cada $\xi \in \mathcal{H}$ é

$$
\begin{aligned}
\gamma(\xi)(\eta) & =f_{\xi}(\eta) \\
& =\langle\xi, \eta\rangle, \quad \forall \eta \in \mathcal{H}
\end{aligned}
$$

em que $\gamma$ é uma isometria antilinear sobrejetora em $\mathcal{H}^{*}$. 
Uma consequência direta da Representação de Riesz é que $\mathcal{H}^{*}$, que é o dual de $\mathcal{H}$, é um espaço de Hilbert com o seguinte produto interno

$$
\left\langle f_{\xi}, f_{\eta}\right\rangle_{\mathcal{H}^{*}}=\langle\eta, \xi\rangle_{\mathcal{H}}, \quad f_{\xi}, f_{\eta} \in \mathcal{H}^{*} .
$$

Vale ressaltar também que o espaço de Hilbert é reflexivo, isto é, o dual do dual é o próprio espaço $\left(\mathcal{H}^{*}\right)^{*}=\mathcal{H}$. Temos também que uma Base Ortonormal de $\mathcal{H}$ é um subconjunto $\left\{\xi_{\alpha}\right\}_{\alpha \in I}$ ortonormal de $\mathcal{H}$, isto é,

$$
\begin{array}{rlrl}
\left\langle\xi_{\alpha}, \xi_{\beta}\right\rangle & =0, & & \text { se } \alpha \neq \beta \\
\left\|\xi_{\alpha}\right\| & =1, & \forall \alpha \in I
\end{array}
$$

tal que $\overline{\operatorname{Lin}\left(\left\{\xi_{\alpha}\right\}_{\alpha \in I}\right)}=\mathcal{H}$, onde $\operatorname{Lin}\left(\left\{\xi_{\alpha}\right\}_{\alpha \in I}\right)$ é o menor espaço vetorial que contém $\left\{\xi_{\alpha}\right\}_{\alpha \in I}$ e a barra simboliza o fecho.

Com isso, todo espaço de Hilbert não trivial (ou seja, $\mathcal{H} \neq 0$ ) possui uma base ortonormal. Como estamos considerando espaços de Hilbert separáveis, essa base é contável. Uma consequência direta deste fato é que uma soma enumerável de espaços de Hilbert também é um espaço de Hilbert, isto é, seja I um conjunto contável e seja $\left\{\mathcal{H}_{i} ; i \in I\right\}$ uma coleção de espaços de Hilbert com produto interno $\langle., . .\rangle_{i}$, então $\bigoplus_{i \in I} \mathcal{H}_{i}:=\left\{\left(\xi_{i}\right)_{i \in I} ; \xi_{i} \in\right.$ $\mathcal{H}_{i}$ e $\sum_{i \in I}\left\|\xi_{i}\right\|^{2}\langle+\infty\}$ é um espaço de Hilbert.

Por fim, comentaremos sobre o resultado que permite a extensão de operadores lineares sobre um espaço de Hilbert. Seja $T \in B(\operatorname{dom}(T), \mathcal{H})$, onde $\operatorname{dom}(T) \subset \mathcal{H}$ é o domínio de $\mathrm{T}$, que deve ser um conjunto denso em $\mathcal{H}$. Então $T$ possui uma única extensão, denotada por $\widetilde{T} \in B(\mathcal{H}, \mathcal{H})$, em que

$$
\left.\widetilde{T}\right|_{\operatorname{dom}(T)}=T .
$$

\subsection{Noções de Teoria Espectral}

A Teoria Espectral baseia-se na análise do espectro dos operadores lineares. Intuitivamente o espectro pode ser pensado como sendo a generalização do conceito de autovalor, essencial no estudo de operadores em espaços de dimensão infinita. Por definição, o Espectro de um operador linear $T \in B(\mathcal{H})$, que denotaremos por $\sigma(T)$, é o conjunto de $\lambda \in \mathbb{C}$ em que $(T-\lambda \mathbf{1})$ não possui inversa contínua, ou seja,

$$
\begin{aligned}
\sigma(T)= & \{\lambda \in \mathbb{C} ;(T-\lambda \mathbf{1}) \\
& \text { não possui inversa contínua }\} .
\end{aligned}
$$

Uma consequência desta definição é que o conjunto dos autovalores de um operador pertence ao espectro. Suponha, por absurdo, que $\lambda$ não pertença ao espectro, então $T-\lambda \mathbf{1}$ possui inversa e como $\lambda$ é autovalor segue que

$$
\begin{array}{rlll}
T \mathbf{v}_{\lambda} & =\lambda \mathbf{v}_{\lambda} & \Rightarrow \\
(T-\lambda \mathbf{1}) \mathbf{v}_{\lambda} & = & 0 & \Rightarrow \\
(T-\lambda \mathbf{1})^{-1}(T-\lambda \mathbf{1}) \mathbf{v}_{\lambda} & = & 0 & \Rightarrow \\
\mathbf{v}_{\lambda} & = & 0, &
\end{array}
$$

o que é um absurdo, já que $\mathbf{v}_{\lambda}$ é um autovetor. De fato, se $\lambda$ é autovalor, $T-\lambda \mathbf{1}$ deixa de ser injetiva, portanto a inversa sequer pode existir. Vale ressaltar que para espaços de dimensão finita, o espectro é exatamente igual ao conjunto dos autovalores.

O complementar do Espectro de um operador $T \in$ $B(\mathcal{H})$ é o Resolvente, $\rho(T)$, que pode ser definido como $\rho(T)=\mathbb{C} \backslash \sigma(T)$, isto é, o conjunto de $\lambda \in \mathbb{C}$ em que $(T-\lambda \mathbf{1})$ possui inversa contínua

$$
\rho(T)=\{\lambda \in \mathbb{C} ;(T-\lambda \mathbf{1}) \text { é inversível e contínuo }\} .
$$

Seja $T \in B(\mathcal{H})$, então o resolvente $\rho(T)$ é um conjunto aberto de $\mathbb{C}$ e, consequentemente, o espectro $\sigma(T)$ um conjunto fechado de $\mathbb{C}$. Além disso, para $\lambda \in \mathbb{C}$, se $|\lambda|>$ $\|T\|$ então $\lambda$ pertence ao resolvente, $\lambda \in \rho(T)$.

Além de ser fechado, o espectro de um operador possui outras propriedades:

- $\sigma(T) \neq \emptyset$.

- $\sigma\left(T^{*}\right)=\sigma(T)^{*}$.

- $\sigma(T)$ é um conjunto compacto.

Como o espectro é compacto, é natural se pensar em qual é a sua extensão; para isso introduziremos a o conceito de Raio Espectral de um operador linear, que notaremos por $r(T)$, como

$$
r(T)=\sup _{\lambda \in \sigma(T)}|\lambda| .
$$

Uma forma operacional de se calcular o raio espectral de um operador é a partir da norma do operador

$$
r(T)=\lim _{n \rightarrow+\infty}\left\|T^{n}\right\|^{1 / n} \leq\|T\|
$$

e tem-se que $\sigma\left(T^{n}\right)=\sigma(T)^{n}$ e $r\left(T^{n}\right)=r(T)^{n}$.

Se $T \in B(\mathcal{H})$ for um operador normal, isto é, $T T^{*}=$ $T^{*} T$, então

$$
r(T)=\|T\|
$$

Como os operadores auto-adjuntos, unitários e positivos são também operadores normais, segue que para esses operadores vale que o raio espectral coincide com a norma do operador.

Para o caso em que $U \in B(\mathcal{H})$ é um operador unitário, $U^{*}=U^{-1}$, então

$$
\sigma(U) \quad \subset \quad\{\lambda \in \mathbb{C} ;|\lambda|=1\}
$$


se $T \in B(\mathcal{H})$ é um operador auto-adjunto então $\sigma(T) \subset \mathbb{R}$ tal que

$$
\sigma(T) \subset[-\|T\|,\|T\|],
$$

além disso, ou $-\|T\|$, ou $\|T\|$, ou ambos pertencem ao espectro.

Vale a pena ressaltar que se $T, V \in B(\mathcal{H})$ forem operadores auto-adjuntos tais que $T V=V T$, então $\mathcal{H}$ possui uma base ortonormal de autovetores simultâneos de $T$ e $V$.

Dentro do contexto da Análise Funcional, um Grupo Unitário a 1-parâmetro Fortemente Contínuo é uma função $U: \mathbb{R} \rightarrow B(\mathcal{H})$ tal que, $\forall s, t \in \mathbb{R}$ temos que

i) $U(t)$ é unitário.

ii) $U(t+s)=U(t) U(s)$

iii) $\lim _{s \rightarrow t}(U(s) \mathbf{v})=U(t) \mathbf{v}$, para todo $\mathbf{v} \in \mathcal{H}$.

terminamos esta subseção com o Teorema de Stone, que será útil para caracterizar o operador de evolução temporal, em que se $U$ é um Grupo Unitário a 1-parâmetro Fortemente Contínuo então existe um operador autoadjunto, $A$, tal que

$$
U(t)=e^{i t A}
$$

\section{3. $C^{*}$-Álgebra e os Teoremas de Gelfand e Gelfand-Neumark}

As $C^{*}$-Álgebras são um tipo particular de Álgebras de Banach, que constituem uma larga área de estudos dentro da Análise Funcional. Nesta área diversos avanços foram feitos, entre eles podemos apontar o Teorema de Gelfand que caracteriza as $C^{*}$-Álgebras abelianas e o Teorema de Gelfand-Neumark que caracteriza as $C^{*}$-Álgebras não abelianas. Um estudo mais detalhado a respeito das $C^{*}$ Álgebras pode ser encontrado nas referências $34 \quad 37$

\section{1. $C^{*}$-Álgebra}

Ao longo de todo o texto, consideraremos aqui uma álgebra $\mathcal{A}$ como sendo um espaço vetorial separável sobre o corpo dos complexos $\mathbb{C}$, munido de uma operação de produto que é bilinear e associativa. Por simplicidade, denotaremos o produto de dois elementos pela justaposição.

Uma Álgebra Normada é uma Álgebra equipada com uma função norma

$$
a \in \mathcal{A} \mapsto\|a\| \in \mathbb{R}
$$

tal que, para todo $a, b \in \mathcal{A}$ e $\lambda \in \mathbb{C}$ temos que:

(i) $\|a\| \geq 0$

(ii) $\|a\|=0 \Leftrightarrow a=0$

(iii) $\|\lambda a\|=|\lambda|\|a\|$

(iv) $\|a+b\| \leq\|a\|+\|b\|$

(v) $\|a b\| \leq\|a\|\|b\|$, os itens $(i)-(i v)$ requeridos pela função norma, garantem que $\mathcal{A}$ seja um espaço normado. Já o item $(v)$ dá uma noção de regularidade do produto.

Um conceito crucial dentro da Análise é o conceito de Completude ou Completicidade, isto é, a propriedade de que toda sequência de Cauchy converge. Nem toda Álgebra Normada é necessariamente completa, como é o caso da álgebra dos polinômios com coeficientes complexos. Consequentemente, faz-se necessário a definir uma Álgebra de Banach como uma Álgebra Normada completa.

Dentro dessa estrutura, podemos introduzir uma outra operação, conhecida como Involução

$$
\text { * : } \mathcal{A} \rightarrow \mathcal{A}
$$

satisfazendo as seguintes propriedades, para todo $a, b \in$ $\mathcal{A}$ e $\lambda \in \mathbb{C}$

(vi) $(a+b)^{*}=a^{*}+b^{*}$

(vii) $(\lambda a)^{*}=\bar{\lambda} a^{*}$

(viii) $(a b)^{*}=b^{*} a^{*}$

(ix) $\left(a^{*}\right)^{*}=a$.

onde $\bar{\lambda}$ é o complexo conjugado de $\lambda$.

Uma $C^{*}$-Álgebra é uma Álgebra de Banach com Involução, tal que

(x) $\left\|a^{*} a\right\|=\|a\|^{2}$.

Dois exemplos de $C^{*}$-Álgebras podem ser vistos a seguir: o exemplo 3.1 é a $C^{*}$-Álgebra associada as funções contínuas num espaço topológico Hausdorf sendo a involução a conjugação da função ponto a ponto, enquanto que o exemplo 3.2 é a $C^{*}$-Álgebra dos operadores lineares sobre um espaço de Hilbert

Exemplo 3.1 Seja X um espaço topológico Hausdorff compacto e $C(X)$ o espaço vetorial de todas as funções continuas $f: X \rightarrow \mathbb{C}$. Adicionando o produto ponto a ponto, isto é,

$$
h(x)=(f g)(x)=f(x) g(x), \quad \forall x \in X
$$

temos uma álgebra. Se além disto definirmos a norma de uma função $f \in C(X)$ por

$$
\|f\|_{\infty}=\sup |f(x)|_{x \in X}
$$

resulta numa Álgebra Normada.

\footnotetext{
${ }^{6}$ Uma topologia de um conjunto $X$ é uma família $\tau$ de subconjuntos de $X$, que são denominados de abertos de $X$, e satisfazem as seguintes propriedades: $i) \emptyset, X \in \tau$; ii) Se $A_{1}, A_{2} \in \tau$ então $A_{1} \bigcap A_{2} \in \tau ;$;ii) Dado uma família arbitrária de abertos $\left(A_{\alpha}\right)_{\alpha \in L}$ então $\bigcup_{\alpha \in L} A_{\alpha} \in \tau$. Um espaço topológico é o par $(X, \tau)$, sendo frequentemente denotado somente por $X$.

${ }^{7}$ Um espaço topológico é dito Hausdorff se é sempre possível separar pontos, ou seja, dados $a, b \in X$, com $a \neq b$, então existem duas vizinhanças $V_{a} \ni a$ e $V_{b} \ni b$ em que $V_{a} \bigcap V_{b}=\emptyset$.
} 
A partir do exemplo 3.1 podemos notar que a Mecânica Clássica é uma $\mathrm{C}^{*}$-Álgebra abeliana, onde o espaço dos estados é representado por $X$, como por exemplo o espaço de fase do sistema, enquanto as observáveis fazem parte de $C(X)$, que são as funções contínuas sobre este espaço 38 .

Exemplo 3.2 Seja $\mathcal{H}$ um espaço de Hilbert complexo e $B(\mathcal{H})$ o conjunto de todos os operadores lineares e contínuos. Esse conjunto é um espaço vetorial e com a composição usual de operadores, a norma do operador

$$
\|T\|=\sup \|T(\xi)\| ; \xi \in \mathcal{H},\|\xi\| \leq 1,
$$

e a involução definida como o adjunto, isto é,

$$
\langle T \xi, \eta\rangle=\left\langle\xi,\left(T^{*}\right) \eta\right\rangle, \quad \forall \xi, \eta \in \mathcal{H}
$$

temos uma $C^{*}$-Álgebra.

Já no exemplo 3.2 observamos que a Mecânica Quântica é uma $C^{*}$-Álgebra não abeliana, com os estados pertencentes ao espaço de Hilbert $\mathcal{H}$ e os observáveis, os operadores auto-adjunto, elementos de $B(\mathcal{H})$ [39 40 .

Algumas propriedades elementares das $C^{*}$-Algebras, são que $1=1^{*},\|1\|=1$ e $\|a\|=\left\|a^{*}\right\|$, para todo $a \in$ $\mathcal{A}$. Esses resultados são válidos para qualquer Álgebra Normada com Involução.

De forma análoga ao que foi visto na Seção 2, podemos introduzir a noção de espectro e resolvente de um operador, que agora é um elemento da $C^{*}$-Álgebra. Da mesma forma, podemos falar de elementos auto-adjuntos $(A=$ $\left.A^{*}\right)$ e de elementos positivos $\left(\sigma(P) \subset \mathbb{R}_{+}=[0,+\infty[)\right.$. Um funcional linear, $f: \mathcal{A} \rightarrow \mathbb{C}$, é dito ser um Funcional Linear Positivo se

$$
f\left(a^{*} a\right) \in \mathbb{R}_{+},
$$

Denotaremos por Estado um funcional linear $\psi: \mathcal{A} \rightarrow$ $\mathbb{C}$, com $\psi \in \mathcal{A}^{*}$, positivo e normalizado, ou seja,

$$
\psi(P) \in \mathbb{R}_{+} \quad \text { e } \psi(1)=1
$$

onde $P$ é um operador positivo.

O conjunto de todos os elementos positivos de $\mathcal{A}$ é denotado por $\mathcal{A}_{+}$, enquanto que o conjunto de todos os elementos auto-adjuntos de $\mathcal{A}$ será $\mathcal{A}_{\mathbb{R}}$. Como todo operador positivo em um espaço complexo é consequentemente auto-adjunto, segue que

$$
\begin{aligned}
\mathcal{A}_{+} & =\left\{A^{2} ; A \in \mathcal{A}_{\mathbb{R}}\right\} \\
& =\left\{B^{*} B ; B \in \mathcal{A}\right\},
\end{aligned}
$$

então o conceito de funcional positivo coincide com o da definição de estado. Temos que cada estado $\psi$ de $\mathcal{A}^{*}$ define um pré-produto intern 8

$$
\langle a, b\rangle:=\psi\left(a^{*} b\right) .
$$

${ }^{8}$ Um pré-produto interno é uma aplicação que tem todas as propriedades do produto interno, menos a que $\langle u, u\rangle=0 \Leftrightarrow u=0$, ou seja, podemos ter elementos não nulos cuja seminorma oriunda de $\langle\cdot, \cdot\rangle$ é nula.
Historicamente o estudo das $\mathrm{C}^{*}$-Álgebras foram baseadas no estudo das álgebra de operadores sobre o espaço de Hilbert. Para fazer a conexão entre a estrutura abstrata das $\mathrm{C}^{*}$-Álgebras e $B(\mathcal{H})$ definimos uma Representação de $\mathcal{A}$ em $\mathcal{H}$ como um *-homomorfismo linear, isto é, um homomorfismo linear

$$
\pi \quad: \quad \mathcal{A} \rightarrow B(\mathcal{H})
$$

tal que

$$
\pi\left(a^{*}\right)=[\pi(a)]^{*}, \quad \forall a \in \mathcal{A} \text {. }
$$

Para $\pi$ uma representação de $\mathcal{A}$ num espaço de Hilbert $\mathcal{H}$ e tomando $\xi \in \mathcal{H}$ normalizado, $\|\xi\|=1$, podemos definir a função $f: \mathcal{A} \rightarrow \mathbb{C}$ por

$$
f_{\xi}=\langle\pi(a) \xi, \xi\rangle, \quad \forall a \in \mathcal{A} .
$$

Por definição $f$ é um funcional linear positivo e devido normalização de $\xi, f$ é um estado. De modo geral, todo vetor do espaço de Hilbert de norma 1 está associado a um estado.

Se $\mathcal{A}$ é uma $C^{*}$-Álgebra e $\pi$ uma representação, então

$$
\|\pi(a)\| \leq\|a\|, \quad \forall a \in \mathcal{A} .
$$

No caso em que $\pi$ é um *-isomorfismo sobre sua imagem, então existe uma inversa e utilizando a expressão (35) para a inversa, chegamos a conclusão que $\pi(A)=\|A\|$.

Uma representação, $\pi$, é dita ser:

- Representação Fiel se $k e r(\pi)=0$.

- Representação Irredutível se $\{0\}$ e $\mathcal{H}$ são os únicos subespaços fechados invariantes sobre $\pi(\mathcal{A})$.

\section{2. $C^{*}$-Álgebra Abeliana}

O objetivo dessa seção é apresentar o Teorema de Gelfand, que afirma que toda $C^{*}$-Álgebra abeliana é isometricamente isomorfa à $C^{*}$-Álgebra das funções contínuas sobre um espaço topológico Hausdorff Compacto. Então, ao longo desta seção, toda $C^{*}$-Álgebra é $\mathcal{A}$ abeliana (isto é, comutativa). Uma consequência importante da comutatividade é que para todo $A \in \mathcal{A}$, temos $\|A\|=r(A)$, onde $r(A)$ é o raio espectral.

Um Funcional Linear Multiplicativo, $\phi: \mathcal{A} \rightarrow \mathbb{C}$, é um *-homomorfismo tal que

$$
\phi(A B)=\phi(A) \phi(B), \quad \forall A, B \in \mathcal{A},
$$

segue diretamente do fato de termos um *-homomorfismos que: $\phi(1)=1, \phi$ é positivo e que $|\phi(A)| \leq\|A\|$, como expressado em 35 . O conjunto de todos os funcionais lineares multiplicativos não nulos será notado por $\widehat{\mathcal{A}}$.

Note que para todo $A \in \mathcal{A}$ com $\|A\|=1, \phi$ um funcional linear multiplicativo, então $|\phi(A)| \leq 1$. De fato, se $\|A\| \leq 1$, então $\left\|A^{n}\right\| \leq\|A\|^{n} \leq 1, \forall n \in \emptyset$. Daí, se por absurdo $|\phi(A)|>1$, teríamos $\left|\phi\left(A^{n}\right)\right|=|\phi(A)|^{n} \rightarrow+\infty$ quando $n \rightarrow \infty$, o que implicaria a descontinuidade de 
$\phi$. De onde concluímos que $\forall \phi \in \widehat{\mathcal{A}}, \frac{|\phi(A)|}{\|A\|} \leq 1$, ou seja, $\widehat{\mathcal{A}}$ é um subconjunto da bola unitária fechada de $\mathcal{A}^{*}$. Considerando a topologia fraca* $\widehat{\mathcal{A}}$ é um espaço topológico Hausdorff compacto

Exemplo 3.3 No contexto em que a $C^{*}$-Álgebra $\mathcal{A}$ é um espaço de funções com domínio métrico compacto $K$, os funcionais lineares multiplicativos, $f$, são exatamente as medidas de Dirac suportadas em algum ponto de $K$

$$
f(g)=\int_{K} g d \delta_{x_{0}},
$$

com $g$ uma função sobre $K$ e $\delta_{x_{0}}$ a função (ou medida) Delta de Dirac suportada em $x_{0} \in K$. Note que, nesse caso, os funcionais lineares multiplicativos estão em bijeção com $K$.

No contexto geral de $C^{*}$-Álgebra abeliana $\mathcal{A}$, inspirados no exemplo modelar acima, mostraremos que esta será isomorfa e isométrica a uma $C^{*}$-Álgebra álgebra de funções em que $\widehat{\mathcal{A}}$ fará as vezes do domínio compacto $K$.

Para obtermos o isomorfismo, é fundamental que os funcionais em $\widehat{\mathcal{A}}$ separem pontos em $\mathcal{A}$, isto é, que dados $A, B \in \mathcal{A}$ exista $\phi \in \widehat{A}$ tal que $\phi(A) \neq \phi(B)$. Ora isso é o mesmo que dizer que $A$ e $B$ estão separados por uma curva de nível (no caso, um hiperplano) de $\phi$. Ora, sabemos que as curvas de nível de um funcional linear são hiperplanos que são um transladados de seu núcleo. Portanto, para conhecer suas curvas nível, precisamos caracterizar os núcleos dos funcionais multiplicativos.

Os elementos de $\widehat{\mathcal{A}}$ são exatamente os funcionais lineares $\phi$ tais que $\phi(1)=1$ e cujos núcleos são ideais maximais de $\mathcal{A}$. Vale a pena ressaltar que se a $C^{*}$-Álgebra não tiver unidade então $\widehat{\mathcal{A}}$ é um espaço topológico Hausdorff Localmente Compacto. Como $\mathcal{A}$ está imerso em $\mathcal{A}^{* *}$, o bidual, podemos definir $\because: \widehat{\mathcal{A}} \rightarrow \mathbb{C}$, isto é, funcionais lineares sobre $\mathcal{A}$ tais que

$$
\ddot{A}(\phi)=\phi(A)
$$

que associa a cada elemento de $\mathcal{A}$ um elemento do bidual. O conjunto de todas essas aplicações, $:: \widehat{\mathcal{A}} \rightarrow \mathbb{C}$, pertencem a $C(\widehat{\mathcal{A}})$, que é o conjunto de todas as funções contínuas definidas sobre o espaço topológico Hausdorff compacto $\widehat{A}^{11}$

A respeito do espectro, um resultado importante é que dado $A \in \mathcal{A}$ então

$$
\sigma(A)=\{\phi(A) ; \phi \in \widehat{\mathcal{A}}\} .
$$

\footnotetext{
${ }^{9}$ Uma topologia $\tau_{1}$ é mais fina que $\tau_{2}$ se $\tau_{1} \subset \tau_{2}$. A topologia fraca é a topologia mais fina definida a partir de abertos no seu dual $X^{*}$, enquanto que a topologia fraca-* requer os abertos no bidual $X^{* *}$. ${ }^{10}$ Pelo Teorema de Alaoglu temos que a bola fechada do dual, $B^{*}=$ $\bar{B}_{\mathcal{N}^{*}}(0 ; 1)$, com a topologia fraca* é um espaço topológico Hausdorff compacto.

${ }^{11}$ Para qualquer espaço Hausdorff compacto $X$, o espaço $C(X)$ das funções contínuas sobre $X$ com a norma do sup é uma Álgebra de Banach, ver Exemplo 3.1
}

Devido ao fato de que $\mathcal{A}$ é comutativa, temos que $\|A\|=$ $r(A), \forall A \in \mathcal{A}$, o que implica que para toda $A \in \mathcal{A}$,

$$
\|A\|=\sup _{\phi \in \widehat{\mathcal{A}}}\{\Phi(A)\} .
$$

Note que, no contexto do nosso exemplo 3.3 em que a $C^{*}$-Álgebra é um espaço de funções contínuas com domínio compacto $K$, o espectro de uma função nesse espaço é exatamente sua imagem.

Devido a essa relação entre o conjunto $\widehat{\mathcal{A}}$ e o espectro, é que $\widehat{\mathcal{A}}$ é chamado de Espectro, que é definido de forma puramente algébrica como o conjunto dos homomorfismos lineares multiplicativos não nulos de $\mathcal{A}$.

A idéia da prova do Teorema de Gelfand é mergulhar a $C^{*}$-Álgebra $\mathcal{A}$ em $C(\widehat{\mathcal{A}})$. Isso é feito codificando cada elemento $A$ de $\mathcal{A}$ através da coleção de valores que ele gera quando aplicamos nele cada funcional $\phi \in \widehat{\mathcal{A}}$. Tal é um argumento clássico em topologia e Análise, muito similar ao mergulho canônico que temos de um espaço de Banach em seu duplo dual. Essa codificação, chamada de Transformada de Gelfand, nos dá uma representação Fiel de $\mathcal{A}$ porque a coleção de funcionais $\widehat{\mathcal{A}}$ separa pontos, isto é, dados dois elementos de $A, B \in \mathcal{A}$, existe um funcional $\phi \in \widehat{\mathcal{A}}$ tal que $\phi(A) \neq \phi(B)$, o que é uma consequência da expressão 40. A Transformada de Gelfand é a função $\kappa: \mathcal{A} \rightarrow C(\widehat{\mathcal{A}})$ tal que

$$
\kappa(A)=\ddot{A}, \quad \ddot{A}(\phi)=\phi(A) .
$$

Segue direto da definição $\kappa(A)(\phi)=\phi(A)$, ou seja, que $\kappa$ é um *-homomorfismo. De fato, $\forall \phi \in \widehat{A}$ tem-se:

$$
\begin{aligned}
\kappa(A+B)(\phi) & =\phi(A+B)=\phi(A)+\phi(B) \\
& =\kappa(A)(\phi)+\kappa(B)(\phi), \\
\kappa(A B)(\phi) & =\phi(A B)=\phi(A) \phi(B) \\
& =\kappa(A)(\phi) \kappa(B)(\phi), \\
\kappa\left(A^{*}\right)(\phi) & =(\phi(A))^{*} \\
& =(\kappa(A)(\phi))^{*},
\end{aligned}
$$

$\operatorname{logo} \kappa(A+B)=\kappa(A)+\kappa(B), \kappa(A B)=\kappa(A) \kappa(B) \mathrm{e}$ $\kappa\left(A^{*}\right)=\kappa(A)^{*}$. Além disso, $\kappa$ é uma contração fraca, isto é, $\kappa(A) \leq\|A\|$. A partir dos conceitos de funcionais multiplicativos e da transformada de Gelfand, somos capazes de enunciar o Teorema de Gelfand, cuja demonstração encontra-se no Apêndice A.

Teorema 3.1 (Gelfand) Seja $\mathcal{A}$ uma $C^{*}$-Álgebra abeliana então existe um espaço Hausdorff compacto $X$ tal que $\mathcal{A}$ é isometricamente isomorfa a $C(X)$.

No teorema de Gelfand o espaço Hausdorff compacto é justamente o conjunto dos funcionais lineares multiplicativos, $\widehat{A}$, enquanto que a isometria isomorfa é determinada pela transformada de Gelfand.

Como foi dito anteriormente, se considerarmos uma $C^{*}$-Álgebra sem unidade, $\widehat{A}=X$ seria um espaço Hausdorff localmente compacto de modo que, $C_{0}(X)$ seria o 
conjunto de todas as funções contínuas que se anulam no infinito, no sentido que para todo $\epsilon<0$ existe um compacto $K \subset X$ tal que $|f(x)|<\epsilon, \forall x \notin K$. Temos que quando $X$ for compacto, $C_{0}(X)=C(X)$. O conjunto $C_{0}(X)$ é um espaço de Banach ${ }^{12}$ e, de forma análoga ao que foi feito anteriormente, pode-se definir a transformada de Gelfand e obter um resultado análogo ao caso em que $\mathcal{A}$ é uma $C^{*}$-Álgebra com unidade: Toda $C^{*}$-Álgebra abeliana sem unidade é isometricamente isomorfa a $C_{0}(X)$.

Para a próxima seção, precisamos do conceito de um Vetor Cíclico de uma representação, isto é, seja $\pi: \mathcal{A} \rightarrow$ $B(\mathcal{H})$ uma representação de uma dada $C^{*}$-Álgebra $\mathcal{A}$, então $\mathbf{x} \in \mathcal{H}$ é um vetor cíclico da representação $\pi$ se o conjunto $\{\pi(A) \mathbf{x} ; A \in \mathcal{A}\}$ é denso em $\mathcal{H}$.

Segue direto da definição de vetor cíclico que, se $\pi$ for uma representação irredutível então todo vetor de $\mathcal{H}$ é cíclico, pois, se existisse $\mathbf{x}_{\mathbf{1}} \in \mathcal{H}$ que não fosse cíclico, então o conjunto $T=\left\{\pi(A) \mathbf{x}_{1} ; A \in \mathcal{A}\right\}$ não seria denso em $\mathcal{H}$; por sua vez o fecho de $T$ seria um subespaço fechado diferente de $\{0\}$ e de $\mathcal{H}$; logo a representação não seria irredutível.

\section{3. $C^{*}$-Álgebra Não-Abeliana}

Vamos agora enunciar um dos grandes Teoremas dentro da teoria das $\mathrm{C}^{*}$-Álgebras, que é a Construção GNS que relaciona a existência de representações sobre o espaço de Hilbert com a existência de estados. Em seguida mencionaremos um outro grande resultado que é o Teorema de Gelfand-Neumark que associa toda $C^{*}$-Álgebra a álgebra de operadores sobre um espaço de Hilbert. Para isso, temos que o conjunto $\mathcal{I}=\left\{B \in \mathcal{A} ; \forall A \in \mathcal{A}, \psi\left(A^{*} B\right)=\right.$ $\langle A, B\rangle=0\}$ onde $\psi$ é um estado, isto é, um funcional linear positivo e normalizado, é um ideal a esquerda de $\mathcal{A}$.

Este resultado associa a cada estado um ideal a esquerda, e pela expressão (31) a esse mesmo estado podemos definir um pré-produto interno; tal fato será útil na demonstração do Teorema de Gelfand-Neumark-Segal, que é apresentada no apêndice B.

Teorema 3.2 (Gelfand-Neumark-Segal) Seja $\mathcal{A}$ uma $C^{*}$-Álgebra e $\psi$ um estado, então existe um espaço de Hilbert $\mathcal{H}_{\psi}$ e uma ${ }^{*}$-representação $\pi_{\psi}: \mathcal{A} \rightarrow B\left(\mathcal{H}_{\psi}\right)$ tal que

i) $\mathcal{H}_{\psi}$ contém um vetor cíclico $\mathbf{x}_{\psi}$.

ii) $\psi(A)=\left\langle\mathbf{x}_{\psi}, \pi_{\psi}(A) \mathbf{x}_{\psi}\right\rangle$.

iii) Qualquer outra representação $\pi$ num espaço $\mathcal{H}_{\pi}$ com vetor cíclico $\mathbf{x}$ é unitariamente equivalente a $\pi_{\psi}$.

A partir da expressão 31 o estado $\psi$ induz um préproduto interno e define um ideal a esquerda $\mathcal{I}$; tomando o quociente $\mathcal{A} / \mathcal{I}$ o pré-produto interno torna-se um produto interno no espaço quociente. Ao completarmos o

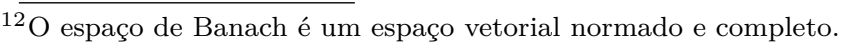

espaço, pela expressão (3), obtemos um espaço de Hilbert que será denotado por $\mathcal{H}_{\psi} \mathrm{O}$ operador $\pi_{\psi}$ é construído a partir de um *-homomorfismo entre $\mathcal{A}$ e $B(\mathcal{A} / \mathcal{I})$, e estendido até $\mathcal{H}_{\psi}$ pela expressão 12 .

As propriedades $i$ ), ii) e $i i i)$ são consequências diretas da construção apresentada no apêndice B. O vetor cíclico é a própria identidade; o estado determina o produto interno por construção e qualquer outra representação é unitariamente equivalente a $\pi_{\psi}$, já que todas são construídas a partir do mesmo estado $\psi$.

Uma consequência muito importante do Teorema 3.2 é a definição de um operador $\rho$, associado ao estado $\psi$, conhecido como Operador Densidade, que são positivos $\operatorname{com} \operatorname{Tr} \rho:=\sum_{n \in \mathbb{N}}\left\langle\mathbf{e}_{n}, \rho \mathbf{e}_{n}\right\rangle=1$ satisfazendo a seguinte relação

$$
\begin{aligned}
\psi(A) & =\left\langle\mathbf{x}_{\psi}, \pi_{\psi}(A) \mathbf{x}_{\psi}\right\rangle \\
& =\operatorname{Tr}\left[\pi_{\psi}(A) \rho\right] .
\end{aligned}
$$

A partir da construção GNS, todo estado define uma realização concreta de uma $C^{*}$-Álgebra na álgebra dos operadores sobre um espaço de Hilbert. Utilizando o Teorema de Gelfand-Neumark-Segal, obtemos que

Teorema 3.3 (Gelfand-Neumark) Toda $C^{*}$-Álgebra é isometricamente isomorfa a uma Álgebra de Operadores sobre um espaço de Hilbert.

Pela construção GNS, para estado $\psi$ temos uma representação $\pi_{\psi}$ e um espaço de Hilbert $\mathcal{H}_{\psi}$. Se definirmos o espaço de Hilbert $\mathcal{H}=\bigoplus_{\psi \in \mathcal{F}} \mathcal{H}_{\psi}$, podemos construir a isometria isomorfa, similar ao caso GNS. Uma consequência muito importante do Teorema 3.3 que é demonstrado no apêndice $\mathrm{B}$, é a relação entre um estado $\psi$ e o operador densidade $\rho$. Dado uma $C^{*}$-Álgebra , então $\psi$ é um estado se e somente se existe $\rho \in B(\mathcal{H})$ tal que $\operatorname{Tr} \rho=1$ e $\psi(A)=\operatorname{Tr}(\rho \pi(A))$.

\section{Mecânica Quântica e as $C^{*}$-Álgebras}

O objetivo desta seção é considerar a Mecânica Clássica como sendo descrita por uma $\mathrm{C}^{*}$-Álgebra e intuir que a prescrição algébrica para os estados e observáveis é preservada. Ao estender essa construção para o caso Quântico a descrição Matemática, via operadores auto-adjuntos sobre um espaço de Hilbert, surge como a única opção devido à não comutatividade entre os observáveis [41 44]. Por fim discutimos brevemente sobre os operadores de Weyl e a $\mathrm{C}^{*}$-Álgebra de Weyl, motivada por esta construção 45.

\subsection{Conexão entre as $C^{*}$-Álgebras Abelianas e a Mecânica Clássica}

A descrição da Mecânica consiste na construção da Cinemática e da Dinâmica. A Cinemática baseia-se na definição do espaço de estados, que é o conjunto de estados admissíveis ao sistema, e do conjunto de observáveis, que 
são basicamente as quantidades que podem ser medidas. Já a Dinâmica caracteriza como o sistema evolui com o tempo. Buscaremos encontrar a relação entre a estrutura abstrata de $C^{*}$-Álgebra com os entes da Mecânica Clássica, isto é, definir os estados e os observáveis em termos dos elementos algébricos da $\mathrm{C}^{*}$-Álgebra.

Dentro do formalismo Hamiltoniano, o estado do sistema é caracterizado pela posição $q=\left(q_{1}, q_{2}, \ldots, q_{n}\right)$ e pelo momento $p=\left(p_{1}, p_{2}, \ldots, p_{n}\right)$, tais que cada estado é representado por um ponto no Espaço de Fase, isto é, $\omega=\left(q_{1}, q_{2}, \ldots, q_{n}, p_{1}, p_{2}, \ldots, p_{n}\right) \in \Gamma$, onde por simplicidade, consideraremos $\Gamma$ como sendo um conjunto compacto; as observáveis são funções reais e contínuas sobre o Espaço de Fase $\Gamma$. Temos então:

\section{Estados $\longleftrightarrow$ Elementos de $\Gamma$. \\ Observáveis $\longleftrightarrow$ Funções contínuas e reais sobre $\Gamma$.}

Pelo Teorema 3.1 de Gelfand, para toda $C^{*}$-Álgebra abeliana existe um espaço Hausdorff compacto $X$ tal que $\mathcal{A}$ é isometricamente isomorfa a $C(X)$. Sendo $\widehat{A}$ o conjunto de funcionais lineares multiplicativos não nulos que de modo geral são funcionais lineares positivos e normalizados; $\widehat{A}$ é um espaço topológico Hausdorff compacto com topologia fraca*. Podemos assim considerar $X=\widehat{A}$ e pelo isomorfismo isométrico, os elementos da $\mathrm{C}^{*}$-Álgebra estão associados às funções contínuas sobre $X$, então as funções reais de $C(X)$ serão exatamente os elementos auto-adjuntos da $\mathrm{C}^{*}$-Álgebra. Com isso temos a correspondência:

\begin{tabular}{|c|c|c|}
\hline Elementos de $X$ & $\longleftrightarrow$ & $\begin{array}{l}\text { Funcionais lineares } \\
\text { positivos e normalizados }\end{array}$ \\
\hline ões reais e & $\longleftrightarrow$ & $\begin{array}{l}\text { Elementos auto-adjunto } \\
\text { da } C^{*} \text {-Álgebra . }\end{array}$ \\
\hline
\end{tabular}

Como observado no exemplo 3.1 a Mecânica Clássica é uma $\mathrm{C}^{*}$-Álgebra abeliana em que $X$ é justamente o espaço de fase $\Gamma$. Portanto, a partir dos conceitos algébricos, podemos postular o que são os estados e os observáveis como elementos de da $\mathrm{C}^{*}$-Álgebra abeliana.

Postulado 4.1 (Estados Clássicos) Os estados de um sistema clássico, são funcionais lineares positivos normalizados da $C^{*}$-Álgebra.

Postulado 4.2 (Observáveis Clássicas) As observáveis de um sistema clássico são exatamente os elementos autoadjuntos da $C^{*}$-Álgebra.

Vale a pena ressaltar que essa caracterização não depende do formalismo utilizado. Se tivéssemos considerado o formalismo Newtoniano, $\Gamma_{1}$ seria o espaço de configuração, em que $\Gamma_{1}=\left(q_{1}, q_{2}, \ldots, q_{n}\right)$. No formalismo Lagrangeano, $\Gamma_{2}$ é um fibrado tangente de $\Gamma_{1}$, dado por
$\Gamma_{2}=\left(q_{1}, q_{2}, \ldots, q_{n}, \dot{q}_{1}, \dot{q}_{2}, \ldots, \dot{q}_{n}\right)$. Já no formalismo Hamiltoniano, $\Gamma$ é um fibrado cotangente de $\Gamma_{1}$, ou seja, $\Gamma=\left(q_{1}, q_{2}, \ldots, q_{n}, p_{1}, p_{2}, \ldots, p_{n}\right)$.

Em todos esses formalismos, o estado do sistema é descrito como sendo um ponto desses espaços, enquanto que as observáveis são funções reais e contínuas sobre sobre esses espaços.

\subsection{Descrição da Mecânica Quântica via $C^{*}$-Álgebra}

A Mecânica Quântica é responsável pela descrição dos fenômenos que ocorrem na escala atômica. A partir desta escala resultados obtidos por uma análise clássica do sistema não concordam com os valores experimentais. Em 1926, W. Heisenberg percebeu que

Princípio 4.3 (Heisenberg) "Quanto mais precisa a posição é determinada, menos precisão se tem sobre o momento naquele instante, e vice-versa."

Portanto, a posição e o momento não são observáveis compatíveis, isto é, se medirmos primeiro a posição e depois o momento, obteremos resultado distinto da medida do momento e depois a posição. Com isso, temos que os observáveis dentro da Mecânica Quântica não comutam necessariamente.

Utilizando a $C^{*}$-Álgebra para descrever a Mecânica, temos que os conceitos de estado e observável não devem depender se a teoria é clássica ou quântica. Deste modo,

Postulado 4.4 (Estados Quânticos) Os estados de um sistema quântico, são funcionais lineares positivos normalizados da $C^{*}$-Álgebra .

Postulado 4.5 (Observáveis Quânticas) Os observáveis de um sistema quântico são exatamente os elementos auto-adjuntos da $C^{*}$-Álgebra .

Devido ao Princípio de Incerteza os observáveis, que são elementos da $\mathrm{C}^{*}$-Álgebra, são não comutativas. Portanto a $C^{*}$-Álgebra que descreve a Mecânica Quântica deve ser necessariamente não abeliana. Dentro da teoria das $\mathrm{C}^{*}$-Álgebras não abelianas, o Teorema 3.3 de GelfandNeumark elucida que toda $C^{*}$-Álgebra não abeliana é isometricamente isomorfa a uma Álgebra de Operadores sobre um espaço de Hilbert. Devido a este teorema, temos que:

Funcionais lineares $\longleftrightarrow$ Vetores normalizados positivos e normalizados de $\mathcal{H}$.

Elementos da $C^{*}$-Álgebra $\longleftrightarrow$ Operadores sobre $\mathcal{H}$.

Com isso, a caracterização da Cinemática Quântica está completa, já que pelas afirmações 4.4, 4.5.

Estados $\longleftrightarrow$ Vetores normalizados de $\mathcal{H}$. Observáveis $\longleftrightarrow$ Operadores auto-adjuntos sobre $\mathcal{H}$. 


\subsection{Estrutura de Weyl}

Na Mecânica Clássica as observáveis possuem além da estrutura de álgebra de funções, uma estrutura adicional de Álgebra de Lie, onde o produto de Lie é dado pelos Parênteses (ou Colchetes) de Poisson [38, 46,

$$
\begin{aligned}
\{f(q, p), g(q, p)\}= & \frac{\partial f(q, p)}{\partial q} \frac{\partial g(q, p)}{\partial p} \\
& -\frac{\partial g(q, p)}{\partial q} \frac{\partial f(q, p)}{\partial p}
\end{aligned}
$$

tal que $\{q, p\}=1$.

Passando agora para a descrição de um sistema quântico, os observáveis são associadas aos operadores auto-adjuntos sobre um espaço de Hilbert, de modo que essa estrutura de Álgebra de Lie atua agora sobre a álgebra de operadores. O produto de Lie é então representado pelo comutador dos operadores, $[A, B]:=A B-B A$, o que implica em

$$
\{q, p\}=1 \longleftrightarrow[q, p]=i \mathbf{1}
$$

sendo $[q, p]=i \mathbf{1}$, a relação canônica de comutação. O fator imaginário $i$ no comutador de $q$ e $p$ garante a consistência, já que se não o tivéssemos chegaríamos a seguinte contradição

$$
\begin{aligned}
\mathbf{1} & =([q, p])^{*} \\
& =p q-q p \\
& =-[q, p] \\
& =-\mathbf{1}
\end{aligned}
$$

Uma consequência direta da relação de comutação é que o espaço de Hilbert que descreve a Mecânica Quântica não pode ter dimensão finita, pois se assim o fosse, os operadores $p$ e $q$ seriam representados por matrizes. Tomando o traço sobre a relação de comutação, chegamos ao seguinte absurdo

$$
\begin{aligned}
\text { in } & =\operatorname{Tr}(i \mathbf{1}) \\
& =\operatorname{Tr}([q, p]) \\
& =\operatorname{Tr}(q p-p q) \\
& =\operatorname{Tr}(q p)-\operatorname{Tr}(p q) \\
& =\operatorname{Tr}(q p)-\operatorname{Tr}(q p) \\
& =0,
\end{aligned}
$$

onde utilizamos somente que o traço é linear e cíclico. Portanto, $\mathcal{H}$ tem que ser um espaço vetorial de dimensão infinita. Partindo novamente da relação de comutação, temos que

$$
\left[q^{n}, p\right]=i n q^{n-1}, \quad \forall n \in \mathbb{N}
$$

onde utilizamos a relação $[A C, B]=A[C, B]+[A, B] C$, válida para quaisquer operadores $A, B$ e $C$. Então,

$$
\begin{aligned}
n\left\|q^{n-1}\right\| & =\left\|i n q^{n-1}\right\| \\
& =\left\|q^{n} p-p q^{n}\right\| \\
& \leq\left\|q^{n} p\right\|+\left\|p q^{n}\right\| \\
& \leq\left\|q^{n}\right\|\|p\|+\|p\|\left\|q^{n}\right\| \\
& =2\|p\|\left\|q^{n}\right\| \Longrightarrow \\
\|q\|\|p\| & \geq \frac{n}{2}, \quad \forall n \in \mathbb{N}
\end{aligned}
$$

consequentemente $\|q\|,\|p\|$ não podem ser finitas conjuntamente, isto é, se $\|q\|$ for finito então para que a igualdade seja sempre satisfeita $\|p\| \rightarrow+\infty$, ou vice versa.

A interpretação deste resultado reside no fato de que, apesar das medidas serem sempre valores finitos, o Princípio de Heisenberg afirma que quanto maior a precisão de $q$ maior será a imprecisão de $p$. Como a priori a precisão não tem um limite fixado, isso se reflete que o operador momento deve ser representado por um operador ilimitado. O mesmo ocorre se trocarmos o momento pela posição.

Essa questão de ordem técnica foi resolvida por $\mathrm{H}$. Weyl 47], que sugeriu considerar operadores da forma

$$
U(\alpha)=e^{i \alpha q} \quad \text { e } \quad V(\beta)=e^{i \beta p},
$$

conhecidos como Operadores de Weyl, que por serem operadores unitários possuem o espectro limitado ao disco de raio 1 . As relações algébricas entre os operadores de Weyl são inferidas a partir das relações de $q$ e $p$. Para isso utilizaremos o lema de Baker-Campbell-Hausdorff para obter as relações entre os operadores de Weyl. A demonstração deste lema pode ser encontrada na referência [40] e consiste em mostrar que se $A, B$ operadores auto-adjuntos sobre um espaço $\mathcal{H}$ em que $[A, B]=\eta \mathbf{1} \operatorname{com} \eta \in \mathbb{C}$. Então ocorre que

$$
e^{i A} e^{i B}=e^{i A+i B-1 / 2 \eta \mathbf{1}}
$$

num domínio $D$ comum a $A, B, A+B$.

Os operadores de Weyl satisfazem as seguintes operações:

i) $U(\alpha) U(\beta)=U(\alpha+\beta)$

ii) $V(\alpha) V(\beta)=V(\alpha+\beta)$

iii) $U(\alpha) V(\beta)=e^{-i \alpha \beta} V(\beta) U(\alpha)$.

A álgebra sobre os complexos formada por elementos abstratos $U(\alpha), V(\beta)$ com $\alpha, \beta \in \mathbb{R}$, satisfazendo as relações dos operadores de Weyl, é chamada de Álgebra de Weyl, que denotaremos por $\mathcal{A}_{W}$.

Dentro da estrutura das Álgebras de Weyl podemos definir uma involução $*: \mathcal{A}_{W} \rightarrow \mathcal{A}_{W}$ tal que

$$
U(\alpha)^{*}=U(-\alpha), \quad V(\beta)^{*}=V(-\beta),
$$

então tanto $U(\alpha)$ quanto $V(\beta)$ são elementos unitários, no sentido que

$$
U(\alpha)^{*} U(\alpha)=\mathbf{1}, \quad V(\beta)^{*} V(\beta)=\mathbf{1}
$$


Com essa involução e o completamento do espaço, as Álgebras de Weyl formam uma $\mathrm{C}^{*}$-Álgebra, onde as normas de $U(\alpha), V(\beta), U(\alpha) V(\beta)$ valem 1 , denominadas $\mathrm{C}^{*}$-Álgebras de Weyl.

No formalismo Hamiltoniano, a evolução temporal da Mecânica Clássica é gerada pelo parêntese de Poisson, que satisfaz uma Álgebra de Lie. Tanto a Mecânica Clássica como a Mecânica Quântica admitem uma estrutura de Álgebra de Lie 48], onde a dinâmica Quântica pode ser obtida a partir do Teorema 21 requerendo que o operador evolução temporal seja um grupo unitário a 1-parâmetro fortemente contínuo; que por sua vez permite encontrar as equações de Schrödinger e de Heisenberg 39,40

\section{Conclusões}

Para analisar a estrutura matemática da Mecânica Quântica utilizamos uma descrição via $\mathrm{C}^{*}$-Álgebra. Essa descrição consiste inicialmente no mapeamento da Mecânica Clássica dentro das $\mathrm{C}^{*}$-Álgebras, caracterizando o que seriam os estados e as observáveis dentro do contexto de $C^{*}$ Álgebra . Em seguida estendemos essa construção para o caso Quântico levando em conta o Princípio de Incerteza.

Para isso partimos do Teorema de Gelfand para $\mathrm{C}^{*}$ Álgebras abelianas. Nesse contexto, os estados de um sistema clássico são justamente os funcionais lineares positivos e normalizados, enquanto que as observáveis clássicas assumem a forma de elementos auto-adjuntos. Portanto, a Mecânica Clássica é descrita por uma $C^{*}$ Álgebra abeliana.

Já na Mecânica Quântica, os resultados experimentais comprovam que os observáveis não comutam necessariamente. Isto acarreta que para a descrição da Mecânica Quântica devemos considerar uma $C^{*}$-Álgebra não abeliana. Portanto, pelo Teorema de Gelfand-Neumark temos que os observáveis agora serão os operadores autoadjuntos sobre um espaço de Hilbert $(\mathcal{H})$ enquanto que os estados são os vetores normalizados dentro deste espaço.

Deste modo, a descrição via $C^{*}$-Álgebra faz com que a Cinemática Quântica surja de forma natural, explicando que a necessidade do espaço de Hilbert na Mecânica Quântica é similar a necessidade de um espaço de Fase na Mecânica Clássica.

A partir da descrição via $C^{*}$-Álgebra , a formulação matemática da Mecânica Quântica surge naturalmente como uma consequência dessa estrutura algébrica e não precisa ser mais postulada da forma que foi feita por $\mathrm{P}$. A. M. Dirac [39].

Uma outra consequência dessa descrição é que podemos observar de forma explícita, a diferença essencial entre as Mecânicas Clássica e Quântica, que é o Princípio de Incerteza, ou seja, o fato dos observáveis não serem necessariamente compatíveis. Podemos inferir que tanto a Mecânica Clássica quanto a Mecânica Quântica são descritas a partir da mesma estrutura matemática, a $C^{*}$-Álgebra, onde as noções de observáveis e estados são as mesmas, sendo a única diferença realmente a não comutatividade.

Portanto, podemos concluir a partir desta descrição que, a passagem do mundo clássico para o mundo quântico é dado pela troca de uma $C^{*}$-Álgebra abeliana por uma $C^{*}$-Álgebra não abeliana. Uma perspectiva do presente trabalho é utilizar a formulação de Schöenberg 4951 da Mecânica Clássica, onde as quantidades dinâmicas são operadores lineares sobre um espaço de HilbertKoopman [48], e analisar a construção GNS nesse contexto clássico.

Os métodos algébricos apresentados aqui também possuem aplicações no contexto da teoria quântica de campos e da mecânica estatística quando o limite termodinâmico é considerado. Existem evidências para a necessidade de uma abordagem algébrica envolvendo um número infinito de graus de liberdade. Conforme salientado por Emch 52. o formalismo de espaço de Fock para campos livres, por exemplo, não é suficiente para uma descrição geral de campos interagentes, a despeito de sua indiscutível utilidade e adequação natural na descrição dos sistemas de muitos corpos.

\section{Material suplementar}

O seguinte material suplementar está disponível online: Apêndice A

Apêndice B

\section{Referências}

[1] J. Von Neumann, Math. Ann. 102, 370 (1929).

[2] J. Von Neumann, Math. Ann. 102, 49 (1929).

[3] F.J. Murray and J. Von Neumann, Ann. of Math. 37, 116 (1936).

[4] F.J. Murray and J. Von Neumann, Trans. Amer. Math. Soc. 41, 208 (1937)

[5] J. Von Neumann, Ann. of Math. 41, 96 (1940).

[6] F.J. Murray and J. Von Neumann, Ann. of Math. 44, 716 (1943).

[7] I. Gelfand, Mat. Sbornik 9, 3 (1941).

[8] I. Gelfand and M. Neumark, Rec. Math. [Mat. Sbornik] 12(54), 197 (1943).

[9] I.E. Segal, Bull. Amer. Math. 53, 73 (1947).

[10] J. Dixmier, $C^{*}$-Algebra (North-Holland Publishing Company, New York, 1969).

[11] R.V. Kadison and J.R. Ringrose, Fundamentals of the Theory of Operator Algebras - Vol. I (Academic Press, New York, 1983).

[12] R.V. Kadison and J.R. Ringrose, Fundamentals of the Theory of Operator Algebras- Vol. II (Academic Press, Orlando, 1986).

[13] R.V. Kadison and J.R. Ringrose, Fundamentals of the Theory of Operator Algebras- Vol. III (Academic Press, New Jersey, 1991).

[14] R.V. Kadison and J.R. Ringrose, Fundamentals of the Theory of Operator Algebras- Vol. IV (Academic Press, New Jersey, 1992). 
[15] O. Bratteli and D. W. Robison, Operator Algebras and Quantum Statistical Mechanics - Vol. I (Springer-Verlag, Berlin, 1987).

[16] O. Bratteli and D. W. Robison, Operator Algebras and Quantum Statistical Mechanics - Vol. II (Springer-Verlag, Berlin, 1997).

[17] R.F. Streater, Physica Scripta 3, 5 (1971).

[18] A. Connes, Noncommutative Geometry (Academic Press, San Diego, 1990).

[19] I. Ojima, Ann. Phys. 137, 1 (1981).

[20] M. Takesaki, Tomita Theory of Modular Hilbert Algebras and its Applications (Springer-Verlag, Berlin, 1970).

[21] A. Matos Neto, J.D.M. Vianna, A.E. Santana and F.C. Khanna, Phys. Essays 9, 596 (1996).

[22] H. Matsumoto, Phys. Essays 9, 583 (1996).

[23] A.E. Santana, A. Matos Neto, J.D.M. Vianna and F.C. Khanna, Int. J. Theor. Phys. 38, 641 (1999).

[24] F.C. Khanna, A.P.C. Malbouisson, J.M.C. Malbouisson and A.E. Santana Thermal Quantum Field Theory: Algebraic Aspects and Applications (World Scientific, Singapore, 2009).

[25] G.G. Emch, Algebraic Methods in Statistical Mechanics and Quantum Field Theory (Wiley-Interscience, New York, 1972).

[26] J.B. Conway, A Course in Functional Analysis (SpringerVerlag, New York, 1985).

[27] C.R. Oliveira, Introdução a Análise Funcional (IMPA, Rio de Janeiro, 2008).

[28] M. Reed and B. Simon, Methods of Modern Mathematical (Academic Press, New York, 1972).

[29] W. Rudin, Functional Analysis (McGraw-Hill, Singapore, 1991).

[30] P. Lax, Functional Analysis (John Wiley \& Sons, New York, 2002).

[31] C. Tomei, Topics in Spectral Theory (IMPA, Rio de Janeiro, 2015).

[32] A.A. de Castro Júnior, Funções de Operador e o Estudo do Espectro (IMPA, Rio de Janeiro, 2013).

[33] R.F. Streater and A.S. Wightman, PCT, Spin and Statistics, and All That (W.A. Beijamin, Inc., New York, 1964).

[34] W. Arveson, An Invitation to $C^{*}$-Algebras (SpringerVerlag, New York, 1976).

[35] K.R. Davidson, C $C^{*}$-algebras by Example (American Mathematical Society, Providence, 1996).

[36] G.J. Murphy, $C^{*}$-algebras and Operator Theory (Academic Press, San Diego, 1990).

[37] R. Exel, Uma Introdução às $C^{*}$-Álgebras, disponível em http://www.mtm.ufsc.br/ exel/papers/ intro.pdf, acessado em 30 de outubro de 2017.

[38] V.I. Arnold, Mathematical Methods of Classical Mechanics (Springer, New York, 1989).

[39] P.A.M. Dirac, Principles of Quantum Mechanics (Oxford University Press, London, 1947).

[40] J.J. Sakurai and J. Napolitano, Modern Quantum Mechanics (Pearson, San Francisco, 2011).

[41] R. Haag, Local Quantum Physics: Fields, Particles, Algebras (Springer, Berlin, 1996).

[42] N.P. Landsman, Lecture Notes on $C^{*}$-Algebras and Quantum Mechanics, disponível em http: //preterhuman.net/texts/science_and_technology/ physics/quantum_mechanics acessado em 30 de outubro de 2017

[43] W. Melo and E. Faria, Mathematical Aspects of Quantum Field Theory (IMPA, Rio de Janeiro, 2007).

[44] F. Strocchi, An Introduction to the Mathematical Structure of Quantum Mechanics (World Scientific, Singapore, 2005).

[45] R.G.G. Amorim, M.C.B. Fernandes, A.R. Queiroz, A.E. Santana w J.D.M. Viana, Rev. Bras. Ens. Fis 35, 3604 (2013).

[46] H. Goldstein, C. Poole and J. Safko, Classical Mechanics (Pearson, San Francisco, 2002).

[47] H. Weyl, The Theory of Groups and Quantum Mechanics (Dover Publication, New York, 1950)

[48] A.E. Santana, A. Mattos Neto e J.D.M. Vianna, Rev. Bras. Ens. Fis 14, 72 (1992).

[49] M. Schöenberg, N. Cimento 9, 1139 (1952).

[50] M. Schöenberg, N. Cimento 10, 419 (1953).

[51] M. Schöenberg, N. Cimento 10, 697 (1953).

[52] G.G. Emch, Mathematical and Conceptual Foundations of 20th Century Physics (Elsevier Science Publishing, Netherlands, 1984). 